\title{
FIXED POINTS OF ISOMETRIES
}

\section{SHOSHICHI KOBAYASHI*}

\section{Statement of Theorem}

The purpose of this paper is to prove the following

Theorem. Let $M$ be a Riemannian manifold of dimension $n$ and let $\xi$ be a Killing vector field (i.e., infinitesimal isometry) of $M$. Let $F$ be the set of points $x$ of $M$ where $\xi$ vanishes and let $F=\cup V_{i}$, where the $V_{i}$ 's are the connected components of $F$. Then (assuming $F$ to be non-empty)

(1) Each $V_{i}$ is a totally geodesic closed submanifold (without singularities) of $M$ and the co-dimension of $V_{i}$ (i.e., $\operatorname{dim} M-\operatorname{dim} V_{i}$ ) is even.

(2) The structure group of the normal bundle over $V_{i}$ can be reduced to $G L(r, C)$, where $2 r$ is the co-dimension of $V_{i}$.

(3) If $x \in V_{i}$ and $y \in V_{j}$ and $i \neq j$, then there is a 1-parameter family of geodesics joining $x$ and $y$ provided $M$ is complete; hence $x$ and $y$ are conjugate to each other.

(4) If $M$ is, moreover, compact, then the Euler number of $M$ is the sum of Euler numbers of $V_{i}$ 's:

$$
\chi(M)=\Sigma \%\left(V_{i}\right)
$$

(the summation is well defined, as the number of connected components $V_{i}$ is finite).

Remarks. (2) implies that if $M$ is orientable, then $V_{i}$ is orientable.

If $F$ consists of only isolated points, then (4) is a particular case of the Index Theorem, as the index of a Killing vector field at an isolated zero point is 1 .

Corollary 1. Let $L$ be an abelian Lie algebra of Killing vector fields of $M$. Let $F$ be the set of points $x$ of $M$ where every element of $L$ vanishes. Then the same statements as in Theorem hold.

Received September 12, 1957.

Revised November 11, 1957.

* This paper was sponsored in part by the National Science Foundation under Grant NSF G-3462, which the author held at the University of Chicago in the summer of 1957. 
Remark. Let $G$ be a torus group acting on a manifold $M$ as a differentiable transformation group. Then we take a Riemannian metric on $M$ invariant under $G$ and apply Corollary 1 , thus obtaining the same results as in Theorem.

Corollary 2. Under the same assumption as in Corollary 1 , if $M$ is a symmetric space in the sense of $E$. Cartan, so is each $V_{i}$.

COROLlary 3. Under the same assumption as in Corollary 1, if the sectional curvature of a complete Riemannian manifold $M$ is non-positive, then $F$ is either empty or connected.

Corollary 4. Let $M$ be a compact manifold of dimension $2 \mathrm{~m}$. Suppose that a torus group of dimension $m$ acts on $M$ (differentiably and effectively). Then the Euler number of $M$ is zero or positive according as the fixed point set $F$ is empty or not. If $M$ is orientable and $F$ is non-empty, then the Euler number of $M$ is greater than or equai to 2.

\section{Proof of Theorem}

(1) Let $x$ be any point of $F$ and let $T_{x}(M)$ be the tangent space to $M$ at $x$. Then $\xi$ induces an endomorphism of $T_{x}(M)$ and it is a skew-symmetric matrix with respect to an orthonormal basis of $T_{x}(M)$. (In classical terminologies, it is an endomorphism defined by the covariant derivatives of $\xi$.) If we choose a proper basis $e_{1}, \ldots, e_{n}$, then this matrix can be reduced to the following

$$
\left(\begin{array}{cccccccccc}
0 & a_{1} & & & & & & & \\
-a_{1} & 0 & & & & & & & & \\
& & & & & & & & \\
& & & 0 & a_{r} & & & \\
& & & -a_{r} & 0 & & & \\
& & & & & 0 & & \\
& & & & & & & & \\
& & & & & & & 0
\end{array}\right)
$$

The 1-parameter group of local isometries generated by $\xi$ induces on $T_{x}(M)$ rotations of the form 


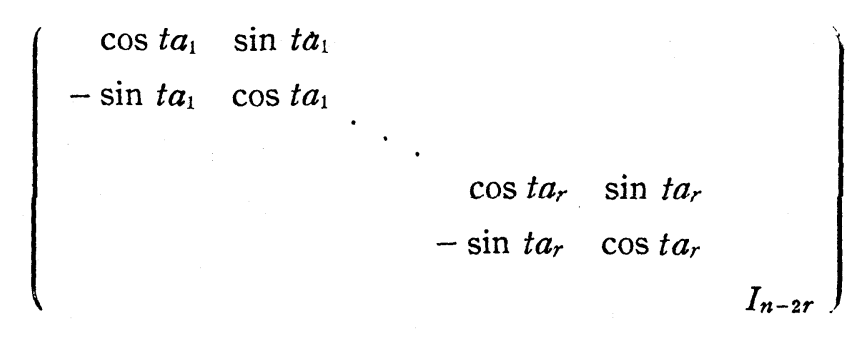

where $t$ is the parameter. If $n-2 r=0$, then $x$ is an isolated zero of $\xi$ and we are done. Suppose $n-2 r>0$. If $v$ is a vector spanned by $e_{2 r+1}, \ldots, e_{n}$, then $v$ is invariant under this 1-parameter group. Hence the geodesic issued from $x$ to the direction of $v$ is also left fixed (pointwise) by the group. In a certain neighborhood $U$ of $x$ the set of these geodesics forms an $(n-2 r)$-dimensional submanifold $U^{\prime}$ of $U$. (Take, for instance, $U$ to be a neighborhood of $x$ such that for every $y$ of $U$ there exists a unique geodesic in $U$ joining $x$ and $y$.) Now we shall show that the zeros of $\xi$ in $U$ are exactly $U^{\prime}$. If $y$ is a zero of $\xi$ in $U$, then we take a geodesic in $U$ joining $y$ and $x$. Since both $x$ and $y$ are left fixed by the 1-parameter group, this geodesic is also left fixed by the group. Hence the tangent vector to this geodesic must be spanned by $e_{2 r+1}, \ldots, e_{n}$. This shows that $y$ is in $U^{\prime}$. Hence each $V_{i}$ is a submanifold of $M$ and its codimension is even. The fact that $V_{i}$ is totally geodesic follows immediately. In fact, let $x$ and $y$ be any points of $V_{i}$ sufficiently close to each other so that there is a unique shortest geodesic from $x$ to $y$. Then this geodesic is left fixed pointwise by the group. Hence the geodesic is contained in $V_{i}$.

Remark. As it can be seen from the proof, the statement that $V_{i}$ is a totally geodesic submanifold of $M$ is true not only for 1-parameter group of isometries but also for any group of isometries.

$(2)^{1)}$ Let $A$ be a non-singular linear transformation of the $2 r$-dimensional vector space $R^{2 r}$ with a positive definite inner product. By the inner product we can identify $A$ with a bilinear form on $R^{2 r}$. Assume that this bilinear form is skew-symmetric. Then there is a unique decomposition of $R^{2 r}$ into subspaces $S_{1}, \ldots, S_{k}$ such that

i) Each $S_{i}$ is invariant by the transformation $A$ and if $i \neq j$ then $S_{i}$ and $S_{j}$ are orthogonal to each other.

1) The result of (2) is due to A. Dold and $R$. Thom. The proof presented here is a modification of theirs. 
ii) Restricted to $S_{i}, A^{2}$ is equal to $-b_{i}^{2} I$, where $I$ is the identity transformation and $b_{i}$ is a positive real number. If $i \neq j$, then $b_{i}$ is different from $b_{j}$.

Let $c_{i}=1 / \sqrt{ } b_{i}$. Let $C$ be a non-singular linear transformation of $R^{2 r}$ defined by the following two properties: (i) $C$ maps each $S_{i}$ into itself, (ii) Restricted on $S_{i}, C$ is equal to $c_{i} I$. Let $J$ be the transformation $C A C$. Then $J^{2}=-I$.

We showed in (1) that the endomorphism of $T_{x}(M)$ induced by $\xi$ induces a non-singular linear transformation, denoted by $A_{x}$, of the normal space to $V_{i}$ at $x$. Since $A_{x}$ is skew symmetric with respect to the inner product on $T_{x}(M)$ defined by the Riemannian metric, we define, by the above argument, a linear transformation $J_{x}$ of $\Gamma_{x}(M)$ such that $J_{x}^{2}=-I$. It can be easily shown that $J_{x}$ is a differentiable field of linear transformations. Now, $J_{x}$ defines a complex structure on each normal space to $V_{i}$; hence the structure group of the normal bundle over $V_{i}$ can be rebuced to $G L(r, C)$.

(3) Let $x \in V_{i}, y \in V_{j}$ and $i \neq j$. Let $g$ be any geodesic from $x$ to $y$. This geodesic can not be left fixed by the group generated by $\xi$. If it were left fixed, then $V_{i}$ and $V_{j}$ would be the same connected component.

(4) Let $\varepsilon$ be a small positive number. We define $S_{x}$ to be the set of points $y$ in $M$ such that there is a geodesic from $x$ to $y$ of the length not greater than $\varepsilon$ and normal to $V_{i}$ at $x$. Thus, to every point $x$ of $V_{i}$, we attach a solid sphere $S_{x}$ with center $x$ and radius $\varepsilon$ which is normal to $V_{i}$ and has the dimension $2 r$ ( = codimension of $V_{i}$ ). Let $N_{i}=\bigcup_{x \in V_{i}} S_{x}$. Taking $\varepsilon$ very small, we may assume that $N_{i} \cap N_{j}$ is empty if $i \neq j$ and that every point in $N_{i}$ is exactly in one $S_{x}$. Let $N=\cup N_{i}$. Let $K$ be the closure of $M-N$. Then $N \cap K$ is the boundary $d N$ of $N$.

LEMma. $\chi(M)=\%(N)+\chi(K)-\chi(d N)$.

Proof. Consider an exact sequence of vector spaces:

$$
\rightarrow A_{k} \rightarrow B_{k} \rightarrow C_{k} \rightarrow A_{k-1} \rightarrow B_{k-1} \rightarrow \ldots
$$

Then it can be shown easily that

$$
\sum(-1)^{k} \operatorname{dim} A_{k}-\sum(-1)^{k} \operatorname{dim} B_{k}+\sum(-1)^{k} \operatorname{dim} C_{k}=0 .
$$

We apply this formula to the exact sequences of homology groups induced by

$$
K \rightarrow M \rightarrow(M, K) \text { and } d N \rightarrow N \rightarrow(N, d N)
$$


and we obtain

$$
\%(K)-\%(M)+\%(M, K)=0 \text { and } \%(d N)-\%(N)+\%(N, d N)=0
$$

By Excision Axiom, $(M, K)$ and $(N, d N)$ have the same relative homology. Hence

$$
\%(M, K)=\%(N, d N)
$$

This completes the proof of Lemma.

The 1-parameter group generated by $\xi$ has no fixed point in $K$ nor $d N$. By Lefschetz Theorem, $\%(K)=\%(d N)=0$. Hence $\%(M)=\%(N)$. As $N_{i}$ is a fibre bundle over $V_{i}$ with solid sphere $S$ as fibre, we have

$$
\varkappa\left(N_{i}\right)=\%\left(V_{i}\right) \%(S)=\%\left(V_{i}\right) .
$$

Finally we obtain

$$
\%(M)=\sum \%\left(N_{i}\right)=\sum \%\left(V_{i}\right)
$$

\section{Proof of Corollaries}

Let $\xi$ and $\eta$ be Killing vector fields on $M$ commuting with each other. Let $F=\cup V_{i}$ be the zeros of $\xi$ as before. Since the group generated by $"$ commutes with the group generated by $\xi$, it maps $F$ into itself. Since it is a connected group, it transforms each $V_{i}$ into itself. Hence $\eta$ can be considered as a Killing vector field on $V_{i}$. Let $F_{i}$ be the zeros of $\eta$ on $V_{i}$ and let $F_{i}=U_{j} W_{i j}$ be the decomposition into the connected components. We apply Theorem to each $V_{t}$ and repeat this process and obtain Corollary 1.

Now, Corollary 2 follows from the fact that every totally geodesic submanifold of a symmetric space is a symmetric space. Note that if $M$ is locally symmetric in the sense that the curvature tensor is parallel, then a simple calculation shows that every totally geodesic submanifold of $M$ is also locally symmetric. Suppose $M$ is globally symmetric. A symmetry of $M$ around any point of a totally geodesic submanifold of $M$ maps the submanifold into itself and induces a symmetry of the submanifold. Hence the submanifold is globally symmetric.

Remark. ${ }^{2}$ It is not known whether the homogeneity of $M$ implies the homogeneity of $V_{i}$.

2) (Added in proof) We shall prove elsewhere that every totally geodesic submanifold of a homogeneous Riemannian manifold is homogeneous Riemannian. 
Corollary 3 follows from (3) and the well known fact that a Riemannian manifold of non-positive curvature has no conjugate points.

Before going into the proof of Corollary 4, we shall make the following

Remark. Suppose that a torus group of dimension $m$ acts on a manifold $M$ of dimension $n$. Assume that the fixed point set $F$ is non-empty. If $2 r$ is the co-dimension of $V_{i}$, then $m \leqq r$.

To prove this, take any Riemannian metric on $M$ invariant by the torus group $G$. Let $x \in V_{i}$. Every element of $G$ induces an orthogonal transformation of $T_{x}(M)$ which is trivial on $T_{x}\left(V_{i}\right)$. Hence $G$ can be considered as a group of orthogonal transformations of the normal space to $V_{i}$ at $x$. $G$ being abelian, $\operatorname{dim} G$ can not be greater than the rank of $0(2 r)$, which is $r$.

The above remark shows than $m \leqq n / 2$. It is therefore of interest to consider the extremal case $2 m=n$. The above argument shows that in this case $F$ consists of only isolated points, thus proving the first half of Corollary 4.

Suppose $M$ is orientable and $F$ consists of a single point $x$. If we take a proper basis of $T_{x}(M)$, the group $G$, considered as a group of orthogonal transformations of $T_{x}(M)$, can be written as follows.

$$
\left(\begin{array}{rrrrr}
\cos t_{1} & \sin t_{1} & & & \\
-\sin t_{1} & \cos t_{1} & & & \\
& & \cdot & & \\
& & & \cos t_{m} & \sin t_{m} \\
& & & -\sin t_{m} & \cos t_{m}
\end{array}\right)
$$

where $\left(t_{1}, \ldots, t_{m}\right)$ is a parameter of $G$. Let $G^{\prime}$ be a torus group of dimension $m-1$ depending on $t_{1}, \ldots, t_{m-1}$. Let $F^{\prime}$ be the fixed point set of $G^{\prime}$ and let $V$ be the connected component of $F^{\prime}$ containing $x$. Then $V$ is a manifold of dimension 2 and is orientable by (2) of Theorem. The 1-parameter group depending on $t_{m}$ maps $V$ into itself. The fixed points of this 1-parameter group on $V$ are in $F=\{x\}$. Hence $\%(V)$ is equal to 1 . On the other hand, the Euler number of a compact orientable surface is always even. This shows that $F$ is either empty or contains more than 1 point.

Institute for Advanced Study, Princeton 